\title{
A NOTE ON THE EXTENSION OF CONTRACTIVE MAPPINGS
}

\author{
J. L. SOLOMON
}

\begin{abstract}
In this note a question posed by J. D. Bryant and L. F. Guseman, Jr. is answered. It is shown that for dense subsets of finite dimensional Banach spaces, the iterative test being conclusive and the contractive extension property are not equivalent. If $(X, d)$ is a complete locally compact and chainable-metric space, a characterization of these dense subsets for which the iterative test is conclusive is given.
\end{abstract}

A self-map $f$ of a metric space $(X, d)$ is said to be contractive if $d(f(x), f(y))<d(x, y)$ for distinct $x, y$ in $X$. M. Edelstein [2] has shown that a contractive self-map $f$ of a metric space $X$ has a fixed point if, for some $x_{0}$, the sequence $\left\{f^{n}\left(x_{0}\right)\right\}$ of iterates at $x_{0}$ contains a subsequence $\left\{f^{n_{i}}\left(x_{0}\right)\right\}$ which converges to $u$; moreover, $u$ is the unique fixed point of $f$ and $\left\{f^{n}\left(x_{0}\right)\right\}$ converges to $u$.

One can easily think of examples such that the only point $x_{0}$ which has a convergent subsequence of iterates is the fixed point itself. Following Nadler [4], we use the following terminology: The iterative test (for contractive maps) is conclusive for $(X, d)$ if and only if for any contractive self-map $f$, the existence of an $x$ in $X$ such that $\left\{f^{n}(x)\right\}$ contains no convergent subsequence allows one to conclude that $f$ has no fixed point. Bryant and Guseman [1] have the following definitions: If $D$ is a dense subset of a metric space $X$ for which each contractive self-map of $D$ has a contractive extension to all of $X$, then $D$ is said to have the contractive extension property; a subset $A$ of a linear space $X$ is called line segment dense if for each $x, y$ in $X$ any line segment (in $X$ ) joining $x$ and $y$ contains a point of $A$ distinct from $x$ and $y$; a subset $S$ of a metric space $(X, d)$ is said to be metric segment dense if for each $x, y$ in $X$, there exists a $z$ in $S$ such that $d(x, z)+d(z, y)=d(x, y)$.

For dense subsets of finite dimensional Banach spaces, Nadler [2] shows that line segment dense implies the contractive extension property which

Received by the editors November 2, 1972.

AMS (MOS) subject classifications (1970). Primary 54C20, 47H10; Secondary 54E40.

Key words and phrases. Fixed point, contractive map, contractive extension property, iterative test. 
implies that the iterative test is conclusive. Bryant and Guseman [1] have shown that being line segment dense and having the iterative test conclusive are not equivalent and show that being metric segment dense implies the contractive extension property. They pose the following (for dense subsets of finite dimensional Banach spaces):

Is the contractive extension property equivalent to the property that the iterative test is conclusive?

The purpose of this note is to answer this question and to provide a characterization of these dense subsets for which the iterative test is conclusive. We shall need the following results of Meyers [3].

THEOREM 1. If $f:(X, d) \rightarrow(X, d)$ is continuous and satisfies: (i) $f\left(x_{0}\right)=x_{0}$ for some $x_{0}$ in $X$, (ii) $\left\{f^{n}(x)\right\}$ converges to $x_{0}$ for each $x$ in $X$, and (iii) there exists an open neighborhood $U$ of $x_{0}$ such that, if $V$ is any neighborhood of $x_{0}$, then there exists a positive integer $n(V)$ such that $f^{n}(U) \subset V$ for each $n \geqq n(V)$; then, for each $\lambda \in(0,1)$, there exists an equivalent metric $d_{\lambda}$ on $X$, complete if $(X, d)$ is complete, such that $f$ is a $d_{\lambda}$-contraction with contraction constant $\lambda$.

Corollary 1. If $(X, d)$ is locally compact, $f:(X, d) \rightarrow(X, d)$ is continuous and satisfies (i) and (ii) of Theorem 1, then the same conclusions are valid.

Definition 1. Let $D$ be a dense subset of a complete metric space $(X, d)$. We say that $D$ has the remetrization contractive extension property (RCEP) if for each contractive $f: D \rightarrow D$ such that $f\left(x_{0}\right)=x_{0}$ for some $x_{0}$ in $D$, then the nonexpansive extension $g: X \rightarrow X$ of $f$ is a contractive map with respect to some equivalent metric on $X$.

THEOREM 2. Let $(X, d)$ be a locally compact metric space which is chainable. Then the iterative test is conclusive for $X$.

Proof. Let $f: X \rightarrow X$ be a contractive self-map with fixed point $x_{0}$. Choose $\varepsilon>0$ such that $\left[S\left(x_{0}, \varepsilon\right)\right]-=\left\{x \in X: d\left(x_{0}, x\right) \leqq \varepsilon\right\}$ is compact. Let $y \in X$. If $y \in\left[S\left(x_{0}, \varepsilon\right)\right]-$, then since $\left[S\left(x_{0}, \varepsilon\right)\right]-$ is compact, $f^{n}(y) \rightarrow x_{0}$. Hence let us assume $y \notin\left[S\left(x_{0}, \varepsilon\right)\right]-$. Since $X$ is chainable, there exists $x_{0}, x_{1}, x_{2}, \cdots, x_{n}=y$ such that $d\left(x_{i}, x_{i+1}\right)<\varepsilon / 2, i=0,1,2, \cdots, n-1$. Let $x_{k}$ be the first element of the chain such that $x_{k} \notin\left[S\left(x_{0}, \varepsilon\right)\right]-$. Thus, for $0 \leqq j \leqq k-1, f^{n}\left(x_{j}\right) \rightarrow x_{0}$ as $n \rightarrow \infty$. There exists a positive integer $N_{k-1}$ such that $d\left(f^{n}\left(x_{k-1}\right), x_{0}\right)<\varepsilon / 2$ for each $n \geqq N_{k-1}$. Since $d\left(x_{k-1}, x_{k}\right)<\varepsilon / 2$ and $f$ is contractive, we have that $f^{N_{k-1}}\left(x_{k}\right)$ is contained in $\left[S\left(x_{0}, \varepsilon\right)\right]-$ and thus $f^{n}\left(x_{k}\right) \rightarrow x_{0}$. By induction we see that $f^{n}(y) \rightarrow x_{0}$ as $n \rightarrow \infty$. Hence $X$ has the iterative test conclusive. 
THEOREM 3. Let $D$ be a dense subset of a complete, locally compact and chainable-metric space $(X, d)$, then the iterative test is conclusive for $D$ if and only if $D$ has the RCEP.

Proof. Assume $D$ has the RCEP. Let $f$ be a contractive self-map on $D$ with fixed point $x_{0}$. Then the extension $g:(X, d) \rightarrow(X, d)$ exists and is nonexpansive. By hypothesis, given $\lambda$ in $(0,1)$, there exists a metric $d_{0}$ equivalent to $d$ such that $g$ is a $d_{0}$ contraction map on $X$. Since $\left(X, d_{0}\right)$ is complete, we have by the Banach contraction mapping theorem that $\left\{g^{n}(x)\right\} \rightarrow x_{0}$ (with respect to $d_{0}$ and thus with respect to $d$ ) for each $x$ in $X$; in particular, $\left\{f^{n}(x)\right\}$ converges to $x_{0}$ for each $x$ in $D$ and the sufficiency is established. To verify the necessity, let $f: D \rightarrow D$ be a contractive map with fixed point $x_{0}$ in $D$. Since $f$ is uniformly continuous and $X$ is complete, the nonexpansive extension $g$ is a self-map of $X$. If $x \in X \sim D$ then, for any sequence $\left\{x_{n}\right\} \subset D$ converging to $x, g(x)=\lim f\left(x_{n}\right)$. Since the iterative test is conclusive for $D$, $\left\{g^{k}(x)\right\}$ converges to $x_{0}$ for each $x$ in $X$; indeed, let $\varepsilon>0$, there exists a positive integer $N$ such that $d\left(x, x_{V}\right)<\varepsilon / 2$ for each $n \geqq N$. The iterative test being conclusive for $D$ insures the existence of a positive integer $k=k\left(x_{N}\right)$ such that $d\left(x_{0}, f^{k}\left(x_{N}\right)\right)<\varepsilon / 2$; whence,

$$
d\left(g^{k}(x), x_{0}\right) \leqq d\left(g^{k}(x), f^{k}\left(x_{.}\right)\right)+d\left(f^{k}\left(x_{N}\right), x_{0}\right)<\varepsilon .
$$

Hence $g^{k}(x) \rightarrow x_{0}$ for each $x$ in $X$ and $g$ is seen to satisfy the conditions of Corollary 1 . Thus for $\lambda$ in $(0,1)$ there exists a metric $d_{\lambda}$ equivalent to $d$ such that $g$ is a $\lambda-d_{\lambda}$-contraction and $D$ has the RCEP.

Example 3. Let $X$ be the plane with the metric induced by the usual Euclidean norm and define $A=\{(x, y) \in X: y=0\} . X$ and $X \sim A$ satisfy the hypothesis of Theorem 2 and thus the iterative test is conclusive. Note also that $X \sim A$ is not metric segment dense (therefore not line segment dense). By Theorem 3, $X \sim A$ has the remetrization contractive extension property. To see that $X \sim A$ does not have the contractive extension property we define a function which is a restriction of a function defined by Nadler [4, Example 3]. Let $(a, b) \in X \sim A$. If $a \neq 0$, define $r(a, b)=\{(x, y) \in X \sim$ $A: y=(2 b / a) x-b$ and $\operatorname{sign}(y)=\operatorname{sign}(b)\}$ and let $r(a, b)$ be the $y$-axis if $a=0$. Then for any $(x, y) \in X \sim A,(x, y)$ is contained in a unique element $r\left(a_{0}, b_{0}\right)$. We define $f(x, y)$ to be the point in $r\left(a_{0}, b_{0}\right)$ with second coordinate $y / 2$. Then $f: X \sim A \rightarrow X \sim A$ is contractive but the extension $g$ of $f$ is not; in fact, each point of $A$ is seen to be a fixed point of $g$. Hence the contractive extension property and the iterative test being conclusive are not equivalent for dense subsets of finite dimensional Banach spaces. 


\section{REFERENCES}

1. Jack Bryant and L. F. Guseman, Jr., Extensions of contractive mappings and Edelstein's iterative test, Canad. Math. Bull. (to appear).

2. M. Edelstein, On fixed and periodic points under contractive mappings, J. London Math. Soc. 37 (1962), 74-79. MR 24 \#A2936.

3. Phillip R. Meyers, A converse to Banach's contraction theorem, J. Res. Nat. Bur. Standards Sect. B. 71B (1967), 73-76. MR 36 \#4521.

4. Sam B. Nadler, Jr., A note on an iterative test of Edelstein, Canad. Math. Bull. 15 (1972), 381-386.

Department of Mathematics, Texas A\&I University, Kingsville, Texas 78363 